\title{
A Modified $H-\alpha$ Plane for the Extraction of Scattering Mechanisms from Dual Circular Polarization SAR Data
}

\author{
Sara Ghods ${ }^{1 *}$, Vahhab Shojaeddini ${ }^{1}$, Yasser Maghsoudi $^{2}$ \\ ${ }^{1}$ Electrical and Information Technology Department of Iranian Research Organization for Science and Technology (IROST), Iran- \\ 1*ghods.sar@gmail.com \\ ${ }^{2}$ Geomatic and Geodesy Department of K.N.Toosi University of Technology, Iran
}

KEY WORDS: Compact polarimetry, Dual Circular Polarisation mode, classification, producer's accuracy, entropy, alpha

\begin{abstract}
:
Cloude-Pottier entropy and $\alpha$-angle are two important parameters for the interpretation of fully polarimetric data. They indicate the randomness of the polarisation of the back scattered waves and the scattering mechanisms of the targets respectively. For fully polarimetric data the $H-\alpha$ plane is presented which using the borders of it the full polarimetric data can be classified into 8 different physical scattering mechanisms. In recent years new approaches have proposed $H$ - $\alpha$ classification spaces by mapping the points which are belong to each PSMs of FP data into the space of $H / \alpha$ for CP data and approximate borders were extracted for the classification purpose. In this paper a novel approach for defining $H / \alpha$ classification plane has been presented which maximizes the producer's accuracy. The optimum borders have been found and the results of classification using the new plane have been compared with the rival method and the superiority of the new proposed method has been revealed.
\end{abstract}

\section{INTRODUCTION}

Nowadays, SAR imaging is a well-developed remote sensing technique for providing high spatial resolution images of the Earth's surface which provides a vast amount of information for environmental monitoring. Fully polarimetric (FP) SAR systems alternately transmit two orthogonal polarizations and receive the response of the scatters to each of them by two antennas with orthogonal polarizations. Transmitting two interleaved electromagnetic waves requires doubling the pulse repetition frequency which implies immediately that the image swath must be only half of the width of a single-polarized or dual-polarized SAR. In order to achieve a better swath width, and coincidentally reduce average power requirements and simplify transmitting hardware, compact polarimetric (CP) systems have been proposed with the promise of being able to maintain many capabilities of fully polarimetric systems (Souyris et al., 2005). One of the most important CP configurations is dual circular polarimetric (DCP) mode.

In order to extract the physical scattering mechanism (PSM) of targets using polarimetric data many classification methods have been presented. One of the most common such methods is $H-\alpha$ decomposition (Cloude and Pottier, 1998) that is proposed for FP data. Its principle relies on the analysis of eigenvalues and eigenvectors of the coherency matrix. The space of scattering entropy $(H)$ and mean alpha angle $(\alpha)$ namely $H-\alpha$ plane is used to classify the image into 8 canonical PSMs.

In recent years two approaches have been proposed in order to find dual $H-\alpha$ classification zones for DCP data. (Guo et al., 2012) proposed an $H-\alpha$ classification space by mapping the points of each PSM from the original FP data into the space of $H-\alpha$ for $\mathrm{CP}$ data and subsequently (Zhang et al., 2014) proposed an $H-\alpha$ space on the basis of the distribution centers and densities of different PSMs. Experimental results showed that the classification accuracy of each PSM is improved compared with the results of Guo's $H-\alpha$ space, however Zhang's method is not well accurate and there are still overlaps between different PSMs.
In this paper an optimum $H-\alpha$ plane is proposed which maximizes the mean producer's accuracy of the classification results for the DCP data simulated from fully polarimetric RADARSAT2 data.

\section{THEORITICAL BACKGROUND}

\subsection{H/a decomposition theory for FP mode}

Remote sensing SARs in FP mode transmit two orthogonal polarizations alternately and record each backscattered wave in two orthogonal polarizations. The scattering matrix represents the FP dataset

$$
S=\left[\begin{array}{ll}
S_{x x} & S_{x y} \\
S_{y x} & S_{y y}
\end{array}\right]
$$

If the radar is monostatic and the reciprocity assumption is valid, then

$S_{x y}=S_{y x}$.

In order to obtain second order statistics and take into account the correlation between neighbor pixels, the target vector $\mathrm{k}$ is defined and from the outer product of the associated target vector with its conjugate transpose the coherency matrix $\mathrm{T}$ is generated.

$$
\begin{aligned}
& k=\frac{1}{\sqrt{2}}\left[\begin{array}{lll}
S_{x x}+S_{y y} & S_{x x}-S_{y y} 2 S_{x y}
\end{array}\right]^{\mathrm{T}} \\
& T=<k \cdot k^{* T}>
\end{aligned}
$$

where the superscript ${ }^{*}$ represents conjugate transpose, and $\langle>$ indicates spatial averaging. Cloude and Pottier have proposed a decomposition method that relies on the analysis of eigenvalue and eigenvector of the coherency matrix 
$T=V\left[\begin{array}{ccc}\lambda_{1} & 0 & 0 \\ 0 & \lambda_{2} & 0 \\ 0 & 0 & \lambda_{3}\end{array}\right] V^{H}$,

where $\lambda_{i}$ is an eigen value and $V$ is the matrix of eigen vectors that is represented by

$V=\left[\begin{array}{lll}v_{1} & v_{2} & v_{3}\end{array}\right], v_{i}$

$=e^{i \varphi}\left[\begin{array}{lll}\cos \alpha_{i} & \sin \alpha_{i} \cos \beta_{i} e^{i \delta_{i}} & \sin \alpha_{i} \sin \beta_{i} e^{i \gamma_{i}}\end{array}\right]^{T}$

in which $v_{i}$ is an eigen vector. Then the polarimetric entropy $\mathrm{H}$ and scattering angle $\alpha$ can be defined as follows:

$$
\begin{aligned}
& \alpha_{i}=\cos ^{-1}\left(\left|v_{1 i}\right|\right) . \\
& \alpha=\sum_{i=1}^{3} p_{i} \alpha_{i} \\
& H=-\sum_{i=1}^{3} p_{i} \log _{2}\left(p_{i}\right) .
\end{aligned}
$$

Where

$$
p_{i}=\lambda_{i} / \sum_{i=1}^{3} \lambda_{i}
$$

Polarimetric $\alpha$ angle relate to the underlying physical scattering mechanisms and is used to associate polarimetric measurements with physical properties of the remotely sensed targets. The concept of entropy in thermodynamic and information theory commonly understood as a measure of uncertainty, similarly in polarimetry the entropy is a measure of randomness in the polarisation of the backscattered wave.

\section{$2.2 \mathrm{H} / \alpha$ decomposition for DCP mode}

The scattering vectors for DCP mode is given by:

$k_{D C P}=\left[\begin{array}{ll}S_{R R} & S_{R L}\end{array}\right]^{T}$

$=\left[\left(S_{H H}-S_{V V}+i 2 S_{H V}\right) i\left(S_{H H}+S_{V V}\right)\right]^{T} / 2$

where the superscript $\mathrm{T}$ denotes the transpose operation. Then, the coherency matrix is as follows:

$T=\left\langle k \cdot k^{*}\right\rangle$.

Eigen decomposition of the coherency matrix is as follows:

$T=V\left[\begin{array}{cc}\lambda_{1} & 0 \\ 0 & \lambda_{2}\end{array}\right] V^{H}, V=\left[v_{1} v_{2}\right]$

in which $\lambda_{1}$ and $\lambda_{2}$ are eigen values and $v_{1}$ and $v_{2}$ are eigen vectors that can be represented as:

$v_{i}=e^{i \varphi}\left[\cos \alpha_{i} e^{i \delta_{i}} \sin \alpha_{i}\right]^{T}, \alpha_{i}=\cos ^{-1}\left(\left|v_{1 i}\right|\right)$.

The scattering entropy and the mean alpha angle can be obtained as follows:

$\alpha=\sum_{i=1}^{2} p_{i} \alpha_{i}$

$H=-\sum_{i=1}^{2} p_{i} \log _{2}\left(p_{i}\right)$.

In which

$$
p_{i}=\frac{\lambda_{i}}{\sum_{i=1}^{2} \lambda_{i}}
$$

\section{PROPOSED METHOD}

When the number of samples in different classes varies greatly, the total accuracy is not a suitable criterion to evaluate the performance of a classifier. So the average producer's accuracy is chosen to find the optimum $H-\alpha$ zones. The producer's accuracy refers to the probability that a certain land-cover of an area on the ground is classified as such. The mean producer accuracy is been calculated as follows:

Mean producer accuracy $=\frac{1}{\mathrm{~N}} \sum_{\mathrm{i}=1}^{\mathrm{N}} \frac{\mathrm{TP}(\mathrm{i})}{\mathrm{n}(\mathrm{i})}$

In which $\mathrm{N}$ is number of classification zones, $\mathrm{n}(\mathrm{i})$ is number of reference pixels in zone(i) and TP(i) is the number of pixels which classified in zone(i) correctly. So the borders of H/a classification planein DCP mode is chosen in such a way that the mean producer accuracy reaches the highest value.

\section{SPECIFICATIONS OF THE DATA SETS}

Two images over San Francisco and Vancouver acquired by Radarsat-2 at C-band in quad polarization mode, with the image size being $1151 \times 1776$ and $1766 \times 1558$ respectively have been used for this study. Google earth images of these areas have been shown in Figure 1.
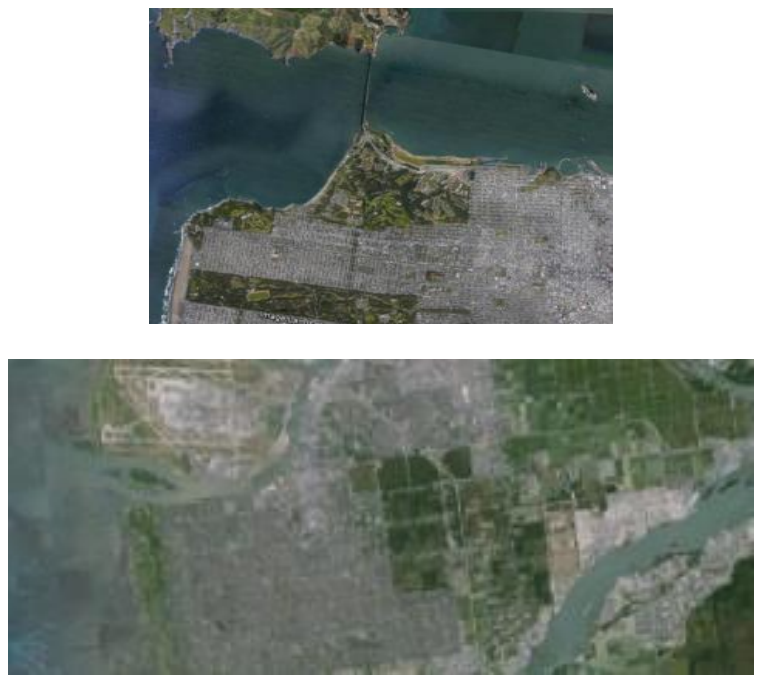

Fig.1. Google earth images of the study areas, San Francisco (Left), Vancouver (Right).

\section{INVESTIGATION OF THE ZHANG'S $H$ - $A$ SPACE}

Figure 2(a) shows the boundaries of different PSMs for the original FP mode. Figure 2(b) shows the distribution of each scattering zones in FP mode which maps to Zhang's $H$ - $\alpha$ space for DCP mode for San Francisco Image. 


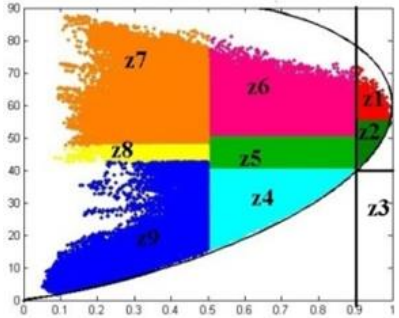

(a)
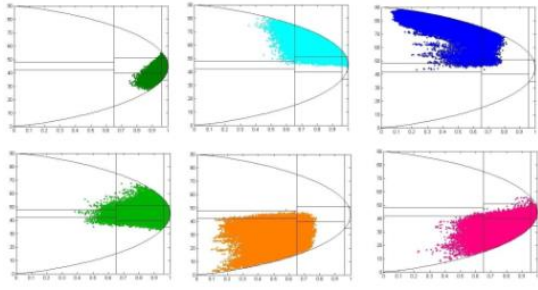

(b)

Fig.2. Boundaries of different PSMs (a) original FP zones, (b) PSMs of FP that are mapped to Zhang's plain.

As it can be seen from Fig.2, Zhang's $H$ - $\alpha$ space for DCP data did not classify PSMs accurately and different PSM's have overlaps with each other in this space. Therefore we tend to find the optimum boundaries for the zones in the $H-\alpha$ space of the DCP data.

\section{OPTIMUM CLASSIFICATION ZONES}

When the number of samples in different classes varies greatly, the total accuracy is not a suitable criterion to evaluate the performance of a classifier. So the average producer's accuracy is chosen to find the optimum $H-\alpha$ zones. The producer's accuracy refers to the probability that a certain land-cover of an area on the ground is classified as such. In figure 3 the boundaries in the $H-\alpha$ plain for DCP data have been named. In figures 4-7 the values of mean producer's accuracy with respect to different values for these boundaries has been plotted.

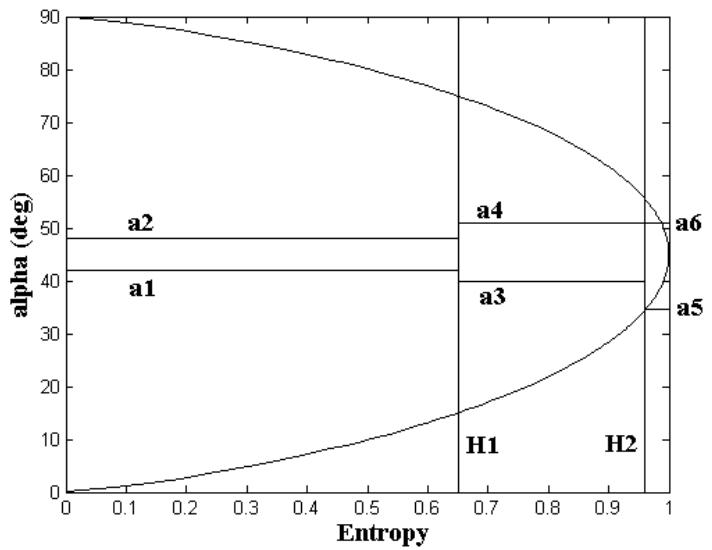

Fig.3. Names of $H-\alpha$ boundaries for classification of different PSMs.

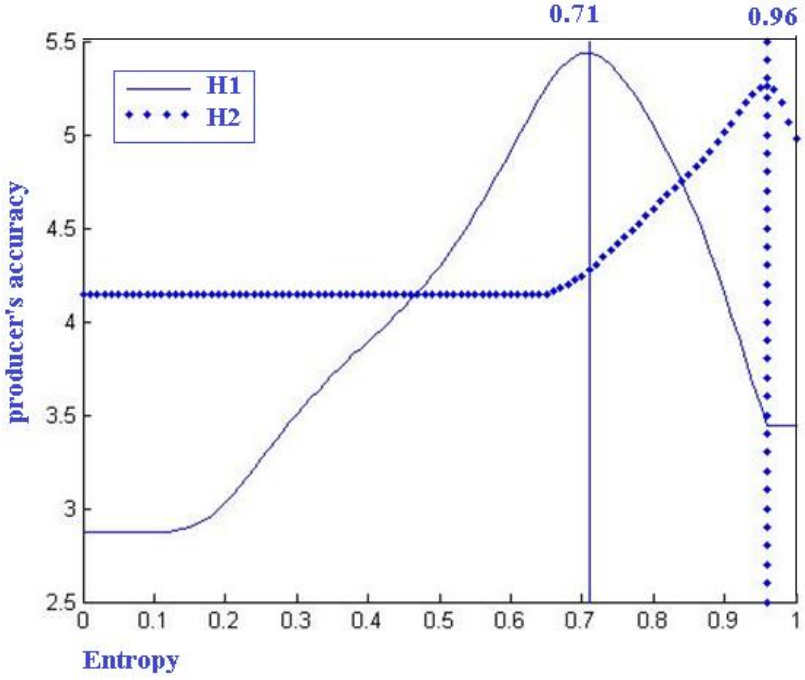

Fig.4. mean producer's accuracy versus different values of the $\mathrm{H} 1$ and $\mathrm{H} 2$ boundaries.

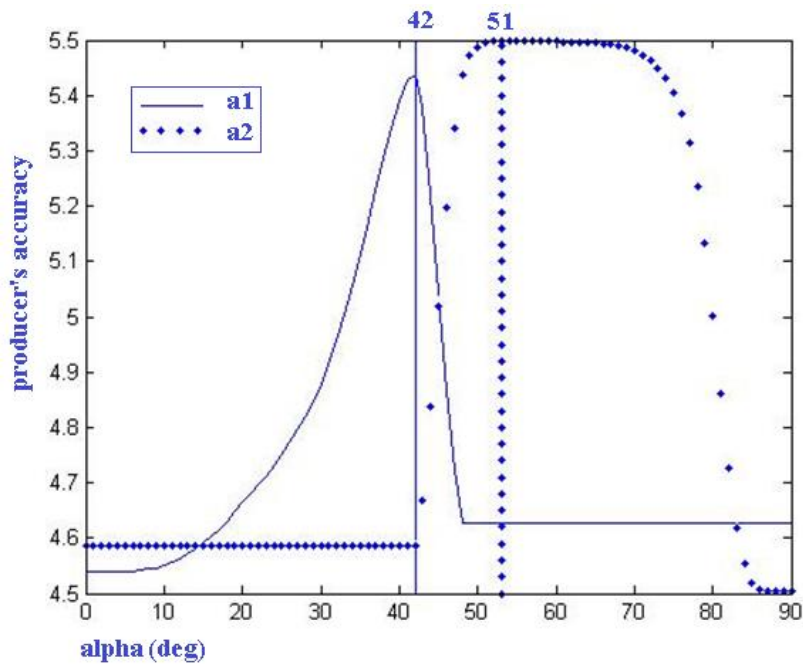

Fig.5. mean producer's accuracy versus different values of the a1 and 2 boundaries.

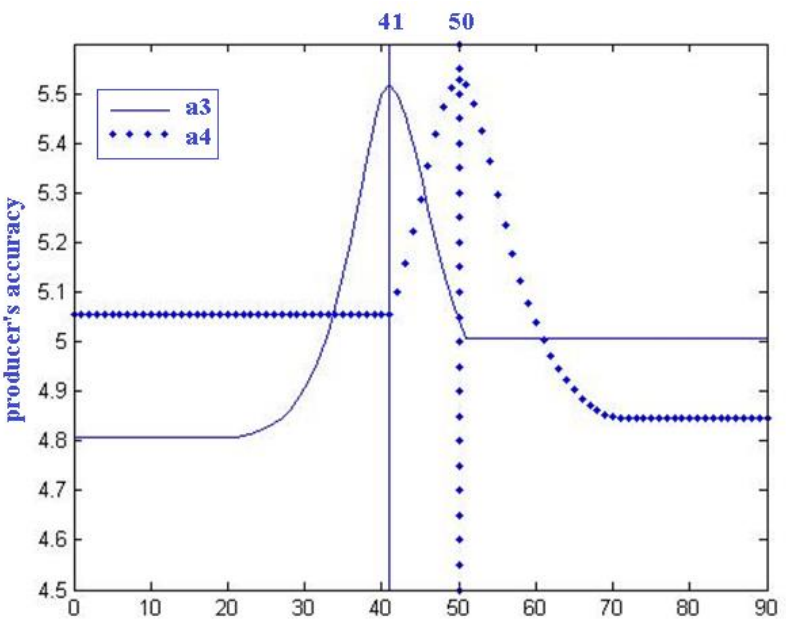

alpha (deg)

Fig.6. mean producer's accuracy versus different values of the $\mathrm{a} 3$ and $\mathrm{a} 4$ boundaries. 


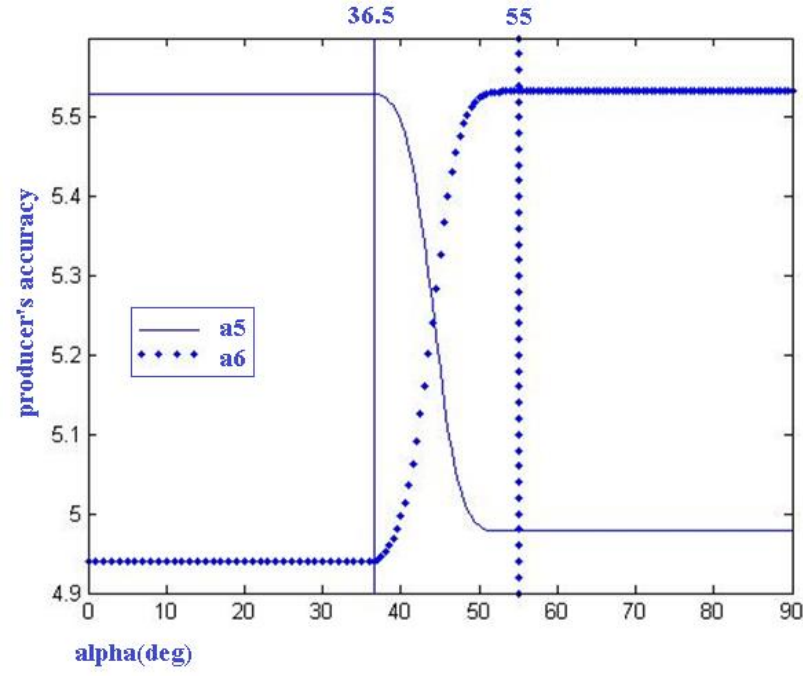

Fig.7. mean producer's accuracy versus different values of the a5 and a6 boundaries.

The resultant optimal values for the parameters which maximize the mean producer's accuracy have been listed in table1.

Table1. Optimal values for $H-\alpha$ classification boundaries.

\begin{tabular}{|l|l|l|l|l|l|l|l|}
\hline H1 & H2 & a1 & a2 & a3 & a4 & a5 & a6 \\
\hline 0.71 & 0.96 & 42 & 53 & 41 & 50 & 36.5 & 55 \\
\hline
\end{tabular}

\section{RESULTS}

In order to evaluate the ability of the proposed $H-\alpha$ zones in comparison with Zhang's zones, Each experimental image is classified into eight PSMs that have been depicted in Figures 5 and 6. Confusion matrices have been achieved and the resultant producer's accuracies have been obtained. Mean producer's accuracies have been calculated which have been presented in table2.

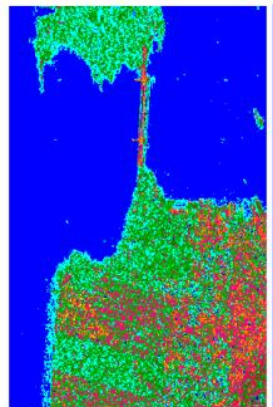

(a)

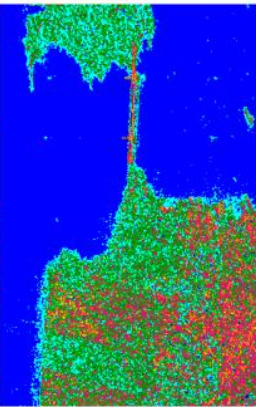

(b)

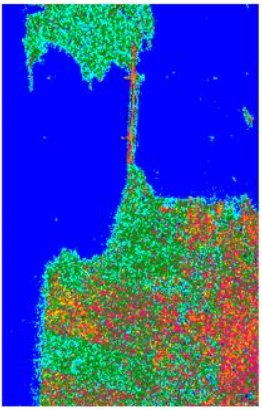

(c)
Fig. 5. classification results for san Francisco data using (a) FP method, (b) Zhang's zones, (c) proposed zones.

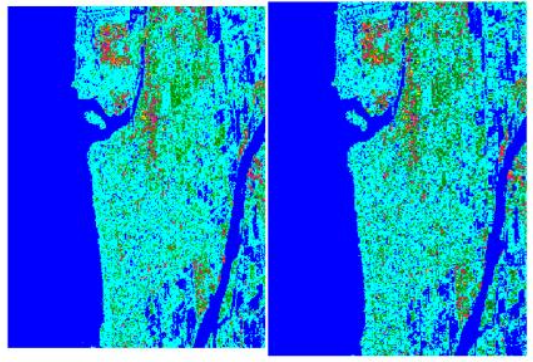

(a) (b)

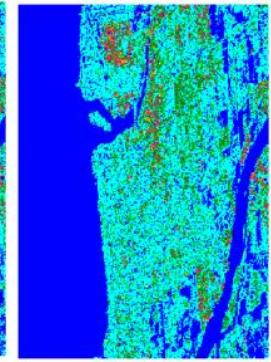

(c)
Fig. 6. classification results for san Francisco data using (a) FP method, (b) Zhang's zones, (c) proposed zones.

Table2. mean producer's accuracies of the classifications based on the proposed and Zhang's $H$ - $\alpha$ zones.

\begin{tabular}{|l|l|l|}
\hline & Zhang's zones & Proposed zones \\
\hline San Francisco & $65.7637 \%$ & $69.1598 \%$ \\
\hline Vancouver & $65.7578 \%$ & $68.0063 \%$ \\
\hline
\end{tabular}

\section{DISCUSSION AND CONCLUSION}

From table 2 the mean producer's accuracy using our proposed method for San Francisco and Vancouver data is $3.4 \%$ and $2.25 \%$ higher than Zhang's method. For the image which for example contained 2044176 pixels, 3\% means that 61325 additional pixels are classified in the original corresponding classes and for the resolution of 5.2*7.6 (m) for RADARSAT2 SLC fine quad mode, it means that additional $2423575 \mathrm{~m}^{2}$ of the scene is classified correctly.

\section{REFERENCES}

Cloude, S. R., Pottier, E., 1997. An entropy based classification scheme for land applications of polarimetric SAR. IEEE Trans. Geosci. Remote Sens., 35(1), pp. 68-78.

Guo, R., Liu, Y. B., Wu, Y. H., Zhang, S. X., Xing, M. D., He, W., 2012. Applying $\mathrm{H} / \alpha$ decomposition to compact polarimetric SAR. IET Radar Sonar Navig., 6(2), pp. 61-70.

Souyris, J. C., Imbo, P., Fjortoft, R., Mingot, S., Lee, J. S., 2005. Compact polarimetry based on symmetry properties of geophysical media: The $\pi / 4$ mode. IEEE Trans. Geosci. Remote Sens., 43(3), pp. 634-646.

Zhang, H., Xie, L., Wang, C., Wu, F., Zhang, B., 2014. Investigation of the Capability of $\mathrm{H}-\alpha$ Decomposition of Compact Polarimetric SAR. IEEE Geoscience and Remote Sensing Letters, 11(4). 